\title{
Stability Analysis of Second Order and Third Order Kind of Tracking Differentiators Based on Lyapunov Method
}

\author{
Wenguang Zhang ${ }^{a}$, Jianting Zheng ${ }^{b}$, Lingdong Zeng ${ }^{c}$ and Junwei Lei ${ }^{\mathrm{a}}$ \\ ${ }^{a}$ College of coastal defense, Naval aviation university, Yantai, 264001, China \\ ${ }^{\mathrm{b}}$ The 92840th Unit of PLA, 572000,China \\ ${ }^{\mathrm{c}}$ The 91515th Unit of PLA , 266400,China
}

Keywords: Stability; Tracking differentiator; Lyapunov function; Analysis; Control system

\begin{abstract}
. two kind of complex tracking differentiators are studied and its stability are proved by constructing Lyapunov function method. And the necessary condition for the input signal is that its derivative should be bounded. And according to the proof process, the stability and design of this kind of tracking differential has no better effect than traditional first order filter method to solve the derivative of a signal.
\end{abstract}

\section{Introduction}

It is very common to use program and computer to solve the derivative of a input signal such as guidance of a target[1-4], we will need the speed information of target to predict the next position of target. So how to use computer and numerical simulation to solve the derivative of a input signal is a very useful question. Many researchers proposed many methods[5-7] to solve this problem, such as traditional first order filter and second order filter method. A kind of tracking differentiator method was proposed and widely studied in the past ten years[8-10]. In this paper, we researched the stability of this kind of tracking differentiator, and we found that, the real effect of this tracking differentiator will be almost the same as traditional first order filter method although no simulation were done to compare its real effect of solving derivative of input signals.

\section{Problem Description}

A kind of second order tracking differentiator can be described as follows:

$$
\begin{aligned}
& \dot{z}_{0}=v_{0} \\
& v_{0}=-\lambda_{0} L^{1 /(n+1)}\left|z_{0}-f(t)\right|^{n /(n+1)} \operatorname{sign}\left(z_{0}-f(t)\right)+z_{1} \\
& \dot{z}_{1}=-\lambda_{1} \operatorname{Lign}\left(z_{1}-v_{0}\right)
\end{aligned}
$$

The Main Problem Is To Prove Its Stability.

\section{Stability Analysis}

Define a new variable as

$$
\varepsilon_{1}=z_{0}-f(t)
$$

Then

$$
\begin{aligned}
& \dot{\varepsilon}_{1}=\dot{z}_{0}-\dot{f}(t)=v_{0}-\dot{f}(t) \\
& =-\lambda_{0} L^{1 /(n+1)}\left|z_{0}-f(t)\right|^{n /(n+1)} \operatorname{sign}\left(z_{0}-f(t)\right)+z_{1}-\dot{f}(t) \\
& =-\lambda_{0} L^{1 /(n+1)}\left|\varepsilon_{1}\right|^{n /(n+1)} \operatorname{sign}\left(\varepsilon_{1}\right)+z_{1}-\dot{f}(t)
\end{aligned}
$$

And

$$
\begin{aligned}
& \dot{z}_{1}=-\lambda_{1} L \operatorname{sign}\left(\lambda_{0} L^{1 /(n+1)}\left|z_{0}-f(t)\right|^{n /(n+1)} \operatorname{sign}\left(z_{0}-f(t)\right)\right) \\
& =-\lambda_{1} L \lambda_{0} L^{1 /(n+1)}\left|z_{0}-f(t)\right|^{n /(n+1)}\left(z_{0}-f(t)\right)
\end{aligned}
$$


Then the whole system can be rewritten as

$$
\begin{gathered}
\dot{\varepsilon}_{1}=-\lambda_{0} L^{1 /(n+1)}\left|\varepsilon_{1}\right|^{n /(n+1)} \operatorname{sign}\left(\varepsilon_{1}\right)+z_{1}-\dot{f}(t) \\
\dot{z}_{1}=-\lambda_{1} L \lambda_{0} L^{1 /(n+1)}\left|\varepsilon_{1}\right|^{n /(n+1)} \varepsilon_{1}
\end{gathered}
$$

Choose a Lyapunov function as

$$
V=\frac{1}{2} z_{1}^{2}+\frac{1}{2} \varepsilon_{1}^{2}
$$

Then its derivative can be solved as

$$
\begin{aligned}
& \dot{V}=\frac{1}{2} z_{1}^{2}+\frac{k}{2} \varepsilon_{1}^{2} \\
& =-\lambda_{0} L^{1 /(n+1)}\left|\varepsilon_{1}\right|^{n /(n+1)}\left|\varepsilon_{1}\right|+\varepsilon_{1} z_{1}-\varepsilon_{1} \dot{f}(t) \\
& -k \lambda_{1} L \lambda_{0} L^{1 /(n+1)}\left|\varepsilon_{1}\right|^{n /(n+1)} z_{1} \varepsilon_{1}
\end{aligned}
$$

If $\dot{f}(t)$ is bounded, then there exist a $k$ such that

$$
\dot{V} \leq=-\lambda_{0} L^{1 /(n+1)}\left|\varepsilon_{1}\right|^{n /(n+1)}\left|\varepsilon_{1}\right|+\left|\varepsilon_{1}\right|
$$

Then if $L$ is big enough, the system is stable, and $\varepsilon_{1}$ is converged to zero.

\section{Third Order Tracking Differentiator}

There a third order tracking differential can be described as follows

$$
\begin{aligned}
& \dot{z}_{0}=v_{0} \\
& v_{0}=-\lambda_{0} L^{1 /(n+1)}\left|z_{0}-f(t)\right|^{n /(n+1)} \operatorname{sign}\left(z_{0}-f(t)\right)+z_{1} \\
& \dot{z}_{1}=v_{1} \\
& v_{1}=-\lambda_{1} L^{1 /(n+1)}\left|z_{1}-v_{0}\right|^{n-1 / n} \operatorname{sign}\left(z_{1}-v_{0}\right)+z_{2} \\
& \dot{z}_{2}=-\lambda_{2} L \operatorname{sign}\left(z_{2}-v_{1}\right)
\end{aligned}
$$

Define a new variable as

$$
\varepsilon_{0}=z_{0}-f(t)
$$

then

$$
\dot{\varepsilon}_{0}=\dot{z}_{0}-\dot{f}(t)=-\lambda_{0} L^{1 /(n+1)}\left|\varepsilon_{0}\right|^{-1 /(n+1)} \varepsilon_{0}+z_{1}-\dot{f}(t)
$$

And

$$
\begin{aligned}
& \dot{z}_{1}=-\lambda_{1} L^{1 /(n+1)}\left|z_{1}-v_{0}\right|^{-1 / n}\left(z_{1}-v_{0}\right)+z_{2} \\
& =-\lambda_{1} L^{1 /(n+1)}\left|\lambda_{0} L^{1 /(n+1)}\right| z_{0}-\left.\left.f(t)\right|^{n /(n+1)} \operatorname{sign}\left(z_{0}-f(t)\right)\right|^{-1 / n} \\
& \left(\lambda_{0} L^{1 /(n+1)}\left|z_{0}-f(t)\right|^{n /(n+1)} \operatorname{sign}\left(z_{0}-f(t)\right)\right)+z_{2} \\
& =-\left.\left.\lambda_{1} L^{1 /(n+1)} \lambda_{0} L^{1 /(n+1)}|| \varepsilon_{0}\right|^{n /(n+1)} \operatorname{sign}\left(\varepsilon_{0}\right)\right|^{-1 / n}\left(\lambda_{0} L^{1 /(n+1)}\left|\varepsilon_{0}\right|^{n /(n+1)} \operatorname{sign}\left(\varepsilon_{0}\right)\right)+z_{2} \\
& =-\lambda_{1} L^{1 /(n+1)} \lambda_{0} L^{1 /(n+1)}\left|\varepsilon_{0}\right|^{-1 /(n+1)} \lambda_{0} L^{1 /(n+1)}\left|\varepsilon_{0}\right|^{-1 /(n+1)} \varepsilon_{0}+z_{2}
\end{aligned}
$$

Then it can rewritten as

$$
\dot{z}_{1}=-\lambda_{1} L^{1 /(n+1)} \lambda_{0} L^{1 /(n+1)}\left|\varepsilon_{0}\right|^{-1 /(n+1)} \lambda_{0} L^{1 /(n+1)}\left|\varepsilon_{0}\right|^{-1 /(n+1)} \varepsilon_{0}+Z_{2}
$$

And the last system can be rewritten as 


$$
\begin{aligned}
& \dot{z}_{2}=-\lambda_{2} \operatorname{Lsign}\left(z_{2}-v_{1}\right) \\
& =-\lambda_{2} L \operatorname{sign}\left(\lambda_{1} L^{1 /(n+1)}\left|z_{1}-v_{0}\right|^{n-1 / n} \operatorname{sign}\left(z_{1}-v_{0}\right)\right) \\
& =-\lambda_{2} L \lambda_{1} L^{1 /(n+1)}\left|z_{1}-v_{0}\right|^{n-1 / n} \operatorname{sign}\left(\operatorname{sign}\left(z_{1}-v_{0}\right)\right) \\
& =-\lambda_{2} L \lambda_{1} L^{1 /(n+1)}\left|z_{1}-v_{0}\right|^{n-1 / n}\left(z_{1}-v_{0}\right) \\
& =-\lambda_{2} L \lambda_{1} L^{1 /(n+1)}\left|z_{1}-v_{0}\right|^{n-1 / n}\left(\lambda_{0} L^{1 /(n+1)}\left|z_{0}-f(t)\right|^{n /(n+1)} \operatorname{sign}\left(z_{0}-f(t)\right)\right) \\
& =-\lambda_{2} L \lambda_{1} L^{1 /(n+1)}\left|z_{1}-v_{0}\right|^{n-1 / n} \lambda_{0} L^{1 /(n+1)}\left|z_{0}-f(t)\right|^{-1 /(n+1)} \varepsilon_{0}
\end{aligned}
$$

It can also be rewritten as

$$
\begin{aligned}
& \dot{z}_{2}=-\lambda_{2} L \operatorname{sign}\left(z_{2}-v_{1}\right) \\
& =-\lambda_{2} L \operatorname{sign}\left(\lambda_{1} L^{1 /(n+1)}\left|z_{1}-v_{0}\right|^{n-1 / n} \operatorname{sign}\left(z_{1}-v_{0}\right)\right) \\
& =-\lambda_{2} L \lambda_{1} L^{1 /(n+1)}\left|z_{1}-v_{0}\right|^{n-1 / n} \operatorname{sign}\left(\operatorname{sign}\left(z_{1}-v_{0}\right)\right) \\
& =-\lambda_{2} L \lambda_{1} L^{1 /(n+1)}\left|z_{1}-v_{0}\right|^{n-1 / n} z_{1}+\lambda_{2} L \lambda_{1} L^{1 /(n+1)}\left|z_{1}-v_{0}\right|^{n-1 / n} v_{0} \\
& =-\lambda_{2} L \lambda_{1} L^{1 /(n+1)}\left|z_{1}-v_{0}\right|^{n-1 / n} z_{1}+\lambda_{2} L \lambda_{1} L^{1 /(n+1)}\left|z_{1}-v_{0}\right|^{n-1 / n} \lambda_{0} L^{1 /(n+1)}\left|\varepsilon_{0}\right|^{-1 /(n+1)} \varepsilon_{0} \\
& -\lambda_{2} L \lambda_{1} L^{1 /(n+1)}\left|z_{1}-v_{0}\right|^{n-1 / n} z_{1}
\end{aligned}
$$

Then the whole system can be rewritten as

$$
\begin{aligned}
& \dot{\varepsilon}_{0}=-\lambda_{0} L^{1 /(n+1)}\left|\varepsilon_{0}\right|^{-1 /(n+1)} \varepsilon_{0}+z_{1}-\dot{f}(t) \\
& \dot{z}_{1}=-\lambda_{1} L^{1 /(n+1)} \lambda_{0} L^{1 /(n+1)}\left|\varepsilon_{0}\right|^{-1 /(n+1)} \lambda_{0} L^{1 /(n+1)}\left|\varepsilon_{0}\right|^{-1 /(n+1)} \varepsilon_{0}+z_{2} \\
& \quad \dot{z}_{2}=-2 \lambda_{2} L \lambda_{1} L^{1 /(n+1)}\left|z_{1}-v_{0}\right|^{(n-1) / n} z_{1}+\lambda_{2} L \lambda_{1} L^{1 /(n+1)}\left|z_{1}-v_{0}\right|^{n-1 / n} \lambda_{0} L^{1 /(n+1)}\left|\varepsilon_{0}\right|^{-1 /(n+1)} \varepsilon_{0}
\end{aligned}
$$

If we choose a Lyapunov function as

$$
V=\frac{1}{2} \varepsilon_{0}^{2}+\frac{k_{1}}{2} z_{1}^{2}+\frac{k_{2}}{2} z_{2}^{2}
$$

Then there exist $k_{1}$ and $k_{2}$ its derivative can be solved as

$$
\begin{aligned}
& \dot{V} \leq-\lambda_{1} L^{1 /(n+1)} \lambda_{0} L^{1 /(n+1)}\left|\varepsilon_{0}\right|^{-1 /(n+1)} \lambda_{0} L^{1 /(n+1)}\left|\varepsilon_{0}\right|^{-1 /(n+1)} \varepsilon_{0}^{2}-\dot{f}(t) \varepsilon_{0} \\
& +\lambda_{2} L \lambda_{1} L^{1 /(n+1)}\left|z_{1}-v_{0}\right|^{n-1 / n} \lambda_{0} L^{1 /(n+1)}\left|\varepsilon_{0}\right|^{-1 /(n+1)} \varepsilon_{0} z_{2}
\end{aligned}
$$

Then if $\dot{f}(t)$ is bounded, the stability of the above tracking differential is proved by Lyapunov stability theorem.

\section{Conclusions}

The stability of second order tracking differentiator and third order differentiator are proved by constructing a Lyapunov function method. And we found that the second order tracking differentiator is easy to prove its stability, also the input signal should be bounded and its derivative also should be bounded, then the stability of above tracking differentiator can be guaranteed.

\section{Reference}

[1] U B.YU F. Design of a Vehicle Lateral Stability Control System via a Fuzzy Logic Control Approach[J].Journal of Automobile Engineering,2010,224 (3):313-326.

[2] Suryanarayanan S, Tomizuk M. Appropriate sensor placement for fault-tolerant lane keeping control of automated vehicles, IEEE/ASME Transactions on Mechatronics,2007,12(4):465-471.

[3]Suryanarayanan S, Tomizuka M, Suzuki T. Design of simultaneously stabilizing controllers and its application to fault. tolerant lane keeping controller design for automated vehicles. IEEE 
transactions on Contro1 Systems Technology, 2004,12(3):329-339.

[4]Li Yinong, Yang Liu, Zheng Ling, et a1. Vehicle longitudinal and lateral coupling control based on sliding mode contro1. China Mechanical Engineering, 2007, 18(7): 866-870.(in Chinese)

[5] Cai L, Rad A B, Chan W L, et a1. Fuzzy PD controller for automatic steering control of autonomous vehicles. Proceedings of the IEEE International Conference on Fuzzy Systems. 2003. 549-554.

[6]Wu S J, Chiang $\mathrm{H} \mathrm{H}$, Perng J W, et a1. The automated lane keeping design for an intelligent vehicle. Proceedings of the IEEE Intelligent Vehicles Symposium. 2005. 508-513.

[7]Oya M, Wang Q. Adaptive lane keeping controller for Four-wheel-steering vehicles, Proceedings of IEEE International Conference on Control and Automation. Guangzhou. 2007. 1942-1947.

[8]Yoshida Y, Wang Q, Oya M, et a1. Adaptive longitudinal velocity and lane keeping control of four-wheel-steering vehicles. SICE Annual Conference. Takamatsu. 2007,1 305- 1310.

[9]Yu X, Man Z. Fast terminal sliding mode control design for nonlinear dynamical systems. IEEE Transactions on Circuits Systems I, 2002, 49(2):261-264.

[10] Wei Li, Jing Wang, Jianming, Self-tuning sliding mode control for vehicle's lane keeping, Journal of Highway and Transportation Research an d Development, 2011, 28(6), 136-140 